\title{
Clinical utility of the nuclear-localized AR-V7 biomarker for treatment choice in metastatic castration-resistant prostate cancer
}

\author{
Virginie Vlaeminck-Guillem ${ }^{1,2} \wedge$ \\ ${ }^{1}$ Centre de Recherche en Cancérologie de Lyon, INSERM 1052 CNRS UMR5286, Centre Léon Berard, Université Claude Bernard Lyon 1, Lyon, \\ France; ${ }^{2}$ Service de Biochimie Biologie Moléculaire Sud, Centre Hospitalier Lyon Sud, Hospices Civils de Lyon, Pierre-Bénite, France \\ Correspondence to: Virginie Vlaeminck-Guillem. Centre de Recherche en Cancérologie de Lyon, INSERM 1052 CNRS UMR5286, Centre Léon \\ Berard, Université Claude Bernard Lyon 1, Lyon, France. Email: virginie.vlaeminck-guillem@univ-lyon1.fr. \\ Comment on: Graf RP, Hullings M, Barnett ES, et al. Clinical Utility of the Nuclear-localized AR-V7 Biomarker in Circulating Tumor Cells in \\ Improving Physician Treatment Choice in Castration-resistant Prostate Cancer. Eur Urol 2020;77:170-7.
}

Submitted Jun 02, 2020. Accepted for publication Jun 19, 2020.

doi: $10.21037 /$ tau-20-968

View this article at: http://dx.doi.org/10.21037/tau-20-968

Metastatic castration-resistant prostate cancer (mCRPC) remains a major concern because of its unavoidable lethality. Current management 'only' aims to prolong survival and the means to fulfill this objective include next-generation androgen receptor signaling inhibitors (ARSIs; abiraterone and enzalutamide and the recently approved apalutamide and darolutamide) and taxanebased chemotherapy (docetaxel, cabazitaxel and paclitaxel). The inevitable evolution of mCRPC to death implies that all patients receiving ASRI or chemotherapy will develop resistance to these agents, whether this resistance is primary (no response at treatment initiation) or secondary (initial response, which becomes exhausted and eventually disappears). When choosing between ARSIs and chemotherapy in clinical practice, there is clearly a tendency to favor ARSIs, presumed to be less toxic than taxanes whereas no randomized trials have been performed to compare taxane-based chemotherapy and ARSI in mCRPC. Some open studies performed comparisons that disclosed either no significant difference between the two regimen or improved outcomes with taxanes (1-6). Specifically after failure of first-line androgen-deprivation therapy, knowing if ARSI or taxane treatment will be more beneficial than the other or even if at least one or the other of these treatments will be effective would be an essential element of the therapeutic choice. In fact there is currently a severe shortage of a marker able to predict the response to these treatments in mCRPC patients. Such a marker, referred as theranostic, would therefore be a useful prerequisite towards personalization of treatment.

A recently published article by Howard Scher's team relaunches the candidate for $\mathrm{AR}-\mathrm{V7}$, a variant of the androgen receptor (AR), as a theranostic marker of mCRPC (7). $\mathrm{AR}-\mathrm{V} 7$ belongs to the growing family of AR variants which are produced from AR gene through structural gene rearrangement and alternative mRNA splicing (8). By contrast with the wild-type full-length AR (AR-FL, which contains four functional regions including the liganddependent transactivation domain and its binding site for natural ligands or antiandrogens), the truncated isoform AR-V7 lacks the C-terminal ligand-binding domain. It is therefore constitutively active: it does not need ligand binding to bind DNA responsive elements in AR target genes (its DNA-binding domain is conserved) and regulate their expression through its conserved $\mathrm{N}$-terminal ligandindependent transactivation domain (8). In other words, $\mathrm{AR}-\mathrm{V} 7$ is able to overcome androgen deprivation and therefore initiate all or part of the AR gene activation program even if androgens are lacking. It is therefore not surprising that its expression in prostate cancer cells has

$\wedge$ ORCID: 0000-0003-2725-7087. 
been soon correlated with poor prognosis $(8,9)$. Even if studies have shown that $\mathrm{AR}-\mathrm{V} 7$ gene activation program is in fact not exactly the same as the AR-FL program, AR-V7 expression became an important molecular mechanism to explain survival of androgen-dependent prostate cancer cells despite androgen deprivation, and consequently failure of castration $(10,11)$. With other molecular mechanisms such as gene amplification, gene mutation or ligand-independent phosphorylation-mediated AR activation, production of truncated AR splicing variants is therefore a witness of the huge AR plasticity developed by tumor cells to overcome androgen deprivation.

The potential value of AR-V7 as a theranostic marker arose from the pioneer work of Antonarakis et al. in 2014: these authors not only suggested that AR-V7 expression is correlated with resistance to enzalutamide and abiraterone, but they also predicted its promising routine use by detecting its expression in circulating tumor cells (CTCs) (12). Biologically-significant circulating tumor materials (cells, DNAs, mRNAs) can indeed be detected in the bloodstream and used as witness of the intrinsic molecular biology of the tumors (13). Such characterization of circulating tumor materials has been called liquid biopsy to underline its ease of use in clinical practice. Using a modified version of the AdnaTest mRNA detection, Antonarakis et al. demonstrated that $39 \%$ and $19 \%$ of 31 enzalutamide- and 31 abiraterone-treated patients had detectable AR-V7 in CTCs, respectively, and that AR-V7-positive CTCs were significantly associated with shorter PSA response rate, PSA progression-free survival, clinical progression-free survival and overall survival (12). Of note, conversion from AR-V7negative status to AR-V7-positive status was observed under enzalutamide or abiraterone treatment, suggesting that the presence of AR-V7 is at least revealed or even induced by these treatments (14). Several studies confirmed the theranostic role of CTC expression of AR-V7 (15), using the AdnaTest mRNA detection (epithelial cell adhesion molecule (EpCAM) enrichment capture followed by ARV7 mRNA detection in pooled CTC aggregates) or variant techniques (16). A significant variation in AR-V7 detection came from the logical demonstration that nuclear AR-V7 expression is more clinically relevant than the sole mRNA identification in whole cells $(17,18)$ : AR-V7 can only exert its transcriptional activity when present in the nucleus and its cytoplasmic presence is likely to be biologically insignificant (19). This detection method uses the pathology slide-based Epic Sciences platform that allows assessment of AR-V7 protein presence and localization in individual
CTCs as traditionally performed in tissue samples (19).

To reinforce the potential value of AR-V7 as a reliable way to guide physician choice in mCRPC, Graf et al. used the Epic Sciences platform (7), as they did previously to disclose an association between the presence of nuclearlocalized AR-V7 protein in CTCs and significant outcomes in patients treated with either ARSIs or taxanes $(18,19)$. In its previous publications, this team showed that patients with nuclear-localized AR-V7 positive CTCs (samples with at least one CTC with a significant nuclear-localized AR-V7) more frequently showed a lack of response with an ASRI. More interestingly, overall survival not only depended on the presence of nuclear-localized ARV7 protein in CTCs but also on the type of treatment: survival was longer in patients with nuclear-localized AR$\mathrm{V} 7$ protein and taxane treatment than in patients with the same AR-V7 distribution and ARSI treatment $(18,19)$. In these studies, patients were however not randomly assigned to either treatment while it is now recognized that a real-world bias associates taxane therapy with more aggressive tumors $(1,3,5)$. In other words it remained to be determined whether the interaction observed between the presence of nuclear-localized AR-V7 protein in CTCs and the treatment specificity $(18,19)$ is biased or not. Since randomized trials, adjusting for confusing variables (such as type of treatment), are not available (ethically irrelevant?), the authors proposed propensity score analyses as a surrogate model (7).

Propensity score analysis aims to address selection bias (as it happens here: ARSI or taxane) when random assignment is not feasible (20). To calculate a propensity score, the probability of assignment to a treatment has to be conditioned by a set of observed covariates. In short, in a (ideally) large cohort of patients receiving either of the treatments, multivariate logistic regression is first performed to associate assignment to a treatment with values specifically taken by interest covariates (see Table 1 for the covariate used in (7)). The obtained regression coefficients are then used to weight individual observations (propensity score weighting) and create new groups with characteristics similar to those created through random assignment. Graf et al. therefore measured physician propensity to choose either an ARSI or a taxane. They confirmed that physicians facing patients with $\mathrm{mCRPC}$ had a propensity to assign them to a taxane rather than an ARSI if the disease is more advanced (higher PSA, liver metastases, high alkaline phosphatase levels, bone metastases...) or if they were administered an ARSI immediately before (7). They also 
Table 1 Factors explored as covariates to be associated with physician decisions to assign either an ARSI or a taxane in patients with $\mathrm{mCRPC}^{*}$

Number of prior systemic therapies in metastatic setting

Prior treatment with ARSI

Prostate-specific antigen levels

Patient's age

Presence of liver metastases

High alkaline phosphatase levels

Presence of bone metastases

Presence of lung metastases

Presence of lymph node metastases

Low albumin levels

High lactate dehydrogenase levels

Low hemoglobin levels

*, factors are here ranked according to the importance of their influence on the choice of taxane treatment (odds for taxane use).

confirmed, after adjusting for physician propensity, that patients with nuclear-localized AR-V7 protein in CTCs had longer overall survival with taxanes over ASRIs. Conversely, AR-V7 negative patients trended to have longer overall survival on ARSIs over taxanes (7). Taken together, their results, obtained through the use of propensity score analysis reinforce the clinical value of the nuclear-localized AR-V7 biomarker in CTCs to guide treatment choice.

Authors of this relevant paper belongs to a team of experts both in the management of mCRPC but also in the use of the Epic Sciences platform. Whether their results would be applicable to all other centers, including those that do not have their expertise remains to be determined. First of all, they used the Epic platform and set the detection of the nuclear presence of AR-V7 as a standard. Many other authors, including pioneers $(10,12,14,16)$, use a standardized AdnaTest mRNA detection method that detects presence of AR-V7 mRNA in the whole CTCs. Although nuclear localization of $\mathrm{AR}-\mathrm{V} 7$ is required for target gene expression control, there is no clear demonstration that nuclear detection is more efficient than whole cell detection in predicting patients' outcome under treatment. Whether AdnaTest mRNA detection would offer the same results is unknown. As stated by the authors themselves, precise ARV7 subcellular localization requires scoring criteria to class CTCs as positive for nuclear AR-V7 staining: at least one CTC with an intact nucleus and nuclear-localized signal- to-noise level above an established background intensity per two pathological slides tested $(7,17-19)$. The authors previously checked the analytical specificity of AR-V7 detection (19): AR-V7-negative and AR-V7-positive tissues as determined by the team's pathologist(s) were included as part of a tissue microarray panel and were confirmed by the microarray evaluation as negative or positive. Whether another pathologist, less experienced in AR-V7 subcellular localization, would have obtained the same results remains to be determined. Another subject of caution is the choice of the covariates used by the expert team to perform their propensity score analysis: whether other teams use the same covariates with the same degree to choose treatment options in mCRPC patients cannot be ascertained. At least, even if cross-resistance is strongly suspected between next-generation ARSIs (apalutamide, darolutamide) and enzalutamide or abiraterone (21), it remains to ascertain whether AR-V7 detection is valuable and reliable before choosing these new ARSIs too.

AR-V7 detection in CTCs is a promising theranostic marker to help treatment decision in mCRPC patients. Although first publications appeared 6 years ago, several points still need clarification such as the detection method (subcellular fractions or whole cells-mRNA detection or antibody-directed protein staining) and the broad applicability as a routine assay in every urological or oncological team. Despite the unavoidable need of confirmation by other studies, the work presented by Graf et al. (7) is highly significant in that it affords solid arguments to consider nuclear AR-V7 as a valuable tool coming in addition to clinical criteria to choose between ARSIs and taxanes in the complex situation of mCRPC. When and how this tool will/would be integrated in valuable guidelines and therefore available in routine practice still remains to be determined.

\section{Acknowledgments}

Funding: None.

\section{Footnote}

Provenance and Peer Review: This article was commissioned by the editorial office, Translational Andrology and Urology. The article did not undergo external peer review.

Conflicts of Interest: The author has completed the ICMJE uniform disclosure form (available at http://dx.doi. 
org/10.21037/tau-20-968). The author has no conflicts of interest to declare.

Ethical Statement: The author is accountable for all aspects of the work in ensuring that questions related to the accuracy or integrity of any part of the work are appropriately investigated and resolved.

Open Access Statement: This is an Open Access article distributed in accordance with the Creative Commons Attribution-NonCommercial-NoDerivs 4.0 International License (CC BY-NC-ND 4.0), which permits the noncommercial replication and distribution of the article with the strict proviso that no changes or edits are made and the original work is properly cited (including links to both the formal publication through the relevant DOI and the license). See: https://creativecommons.org/licenses/by-nc-nd/4.0/.

\section{References}

1. Oh WK, Cheng WY, Miao R, et al. Real-world outcomes in patients with metastatic castration-resistant prostate cancer receiving second-line chemotherapy versus an alternative androgen receptor-targeted agent (ARTA) following early progression on a first-line ARTA in a US community oncology setting. Urol Oncol 2018;36:500.e1-e9.

2. Sonpavde G, Huang A, Wang L, et al. Taxane chemotherapy vs antiandrogen agents as first-line therapy for metastatic castration-resistant prostate cancer. BJU Int 2018;121:871-9.

3. Oh WK, Miao R, Vekeman F, et al. Real-world Characteristics and Outcomes of Patients With Metastatic Castration-resistant Prostate Cancer Receiving Chemotherapy Versus Androgen Receptor-targeted Therapy After Failure of First-line Androgen Receptortargeted Therapy in the Community Setting. Clin Genitourin Cancer 2017;S1558-7673(17)30170-2. [Epub ahead of print].

4. George DJ, Sartor O, Miller K, et al. Treatment Patterns and Outcomes in Patients With Metastatic Castrationresistant Prostate Cancer in a Real-world Clinical Practice Setting in the United States. Clin Genitourin Cancer 2020;S1558-7673(19)30392-1. [Epub ahead of print].

5. Miyake H, Sugiyama T, Aki R, et al. Comparison of Alternative Androgen Receptor-axis-targeted Agent (ARATA) and Docetaxel as Second-line Therapy for Patients With Metastatic Castration-resistant Prostate Cancer With Progression After Initial ARATA in Real- world Clinical Practice in Japan. Clin Genitourin Cancer 2018;16:219-25.

6. Matsubara N, Yamada Y, Tabata KI, et al. Comparison of Sequential Treatment With Androgen Receptor-Targeted Agent Followed by Another Androgen Receptor-Targeted Agent Versus Androgen Receptor-Targeted Agent Followed by Docetaxel in Chemotherapy-Naive Patients With Metastatic Castration-Resistant Prostate Cancer. Clin Genitourin Cancer 2017;15:e1073-80.

7. Graf RP, Hullings M, Barnett ES, et al. Clinical Utility of the Nuclear-localized AR-V7 Biomarker in Circulating Tumor Cells in Improving Physician Treatment Choice in Castration-resistant Prostate Cancer. Eur Urol 2020;77:170-7.

8. Hu R, Dunn TA, Wei S, et al. Ligand-independent androgen receptor variants derived from splicing of cryptic exons signify hormone-refractory prostate cancer. Cancer Res 2009;69:16-22.

9. Guo Z, Yang X, Sun F, et al. A novel androgen receptor splice variant is up-regulated during prostate cancer progression and promotes androgen depletion-resistant growth. Cancer Res 2009;69:2305-13.

10. Antonarakis ES. Predicting treatment response in castration-resistant prostate cancer: could androgen receptor variant-7 hold the key? Expert Rev Anticancer Ther 2015;15:143-5.

11. Sun S, Sprenger CC, Vessella RL, et al. Castration resistance in human prostate cancer is conferred by a frequently occurring androgen receptor splice variant. J Clin Invest 2010;120:2715-30.

12. Antonarakis ES, Lu C, Wang H, et al. AR-V7 and resistance to enzalutamide and abiraterone in prostate cancer. N Engl J Med 2014;371:1028-38.

13. Vlaeminck-Guillem V. When Prostate Cancer Circulates in the Bloodstream. Diagnostics (Basel) 2015;5:428-74.

14. Steinestel J, Luedeke M, Arndt A, et al. Detecting predictive androgen receptor modifications in circulating prostate cancer cells. Oncotarget 2015;10:4213-23.

15. Lu C, Brown LC, Antonarakis ES, et al. Androgen receptor variant-driven prostate cancer II: advances in laboratory investigations. Prostate Cancer Prostatic Dis 2020;23:381-97.

16. Maillet D, Allioli N, Peron J, et al. Improved Androgen Receptor Splice Variant 7 Detection Using a Highly Sensitive Assay to Predict Resistance to Abiraterone or Enzalutamide in Metastatic Prostate Cancer Patients. Eur Urol Oncol 2019;S2588-9311(19)30136-1. [Epub ahead of print].

17. Scher HI, Graf RP, Schreiber NA, et al. Nuclear-specific 
AR-V7 Protein Localization is Necessary to Guide Treatment Selection in Metastatic Castration-resistant Prostate Cancer. Eur Urol 2017;71:874-82.

18. Scher HI, Graf RP, Schreiber NA, et al. Assessment of the Validity of Nuclear-Localized Androgen Receptor Splice Variant 7 in Circulating Tumor Cells as a Predictive Biomarker for Castration-Resistant Prostate Cancer. JAMA Oncol 2018;4:1179-86.

19. Scher HI, Lu D, Schreiber NA, et al. Association of ARV7 on Circulating Tumor Cells as a Treatment-Specific

Cite this article as: Vlaeminck-Guillem V. Clinical utility of the nuclear-localized AR-V7 biomarker for treatment choice in metastatic castration-resistant prostate cancer. Transl Androl Urol 2020;9(6):2483-2487. doi: 10.21037/tau-20-968
Biomarker With Outcomes and Survival in CastrationResistant Prostate Cancer. JAMA Oncol 2016;2:1441-9.

20. Olmos A, Govindasamy P. A Practical Guide for Using Propensity Score Weighting in R. Practical Assessment, Research \& Evaluation 2015;20. Available online: https:// scholarworks.umass.edu/pare/vol20/iss1/13.

21. Zhao J, Ning S, Lou W, et al. Cross-resistance among next generation anti-androgen drugs through the AKR1C3/ AR-V7 axis in advanced prostate cancer. Mol Cancer Ther 2020. doi: 10.1158/1535-7163.MCT-20-0015. 